\title{
Midkine, heparin-binding growth factor, blocks kainic acid-induced seizure and neuronal cell death in mouse hippocampus
}

\author{
Yun B Kim¹,2, Jae K Ryư ${ }^{+1}$, Hong J Lee ${ }^{1,3}$, In J Lim4, Dongsun Park2, Min C Lee 1,5 and Seung U Kim*1,3
}

\begin{abstract}
Background: Midkine (MK), a member of the heparin-binding growth factor family, which includes MK and pleiotrophin, is known to possess neurotrophic and neuroprotective properties in the central nervous system. Previous studies have shown that MK is an effective neuroprotective agent in reducing retinal degeneration caused by excessive light and decreasing hippocampal neuronal death in ischemic gerbil brain. The present study was undertaken to investigate whether MK acts as an anticonvulsant in kainic acid (KA)-induced seizure in mouse and blocks KA-mediated neuronal cell death in hippocampus.
\end{abstract}

Results: Increased expression of MK was found in hippocampus of mouse following seizures induced by intracerebroventricular injection of KA, and MK expression was found in glial fibrillary acidic protein (GFAP)-positive astrocytes. Concurrent injection of MK and KA attenuated KA-induced seizure activity and cell death of hippocampal neurons including pyramidal cells and glutamic acid decarboxylase 67 (GAD67)-positive GABAergic interneurons in the CA3 and hilar area.

Conclusion: The results of the present study indicate that MK functions as an anticonvulsant and neuroprotective agent in hippocampus during KA-induced seizures.

\section{Background}

Temporal lobe epilepsy (TLE) is pathologically characterized by extensive neuronal loss in the CA1, CA3 and hilar regions of hippocampus [1,2]. Previous studies have demonstrated that the animal models of TLE generated by intracerebroventricular injection of kainic acid (KA) faithfully reproduce clinical and pathological features found in human TLE [3-7].

Previous studies have reported the possible involvement of neurotrophic factors in epilepsy as suggested by the gene expression of neurotrophic factors such as NGF, BDNF and NT-3 in hippocampus in human TLE as well as in TLE animal models [8,9]. Midkine (MK), one of such neurotrophic factors, has emerged as an important neuromodulator in the central nervous system (CNS). MK, a member of the heparin-binding growth factor family, which includes MK and

\footnotetext{
* Correspondence: sukim@interchange.ubc.ca

1 Division of Neurology, Department of Medicine, UBC Hospital, University of British Columbia, Vancouver, Canada

+ Contributed equally

Full list of author information is available at the end of the article
}

pleiotrophin, is known to possess neurotrophic and neuroprotective properties $[10,11]$. MK was originally isolated as the product of retinoic acid-responsive gene that functions primarily in inducing cell differentiation in mouse teratocarcinoma cells [12], and has the ability to influence a variety of neuronal functions including neurite extension [13], neuronal differentiation $[14,15]$ and neuronal survival following injury or damage in the CNS $[15,16]$. During the fetal development of the CNS, MK expression was demonstrated in neuroepithelial/neural progenitor cells following ethylnitrosourea injury [17] indicating that MK might have a role in cellular proliferation [18]. Recent studies further showed that MK has been implicated in neurological diseases, including Alzheimer's disease [19], cerebral ischemia [20] and Parkinson-dementia complex of Guam (Lyticobodig disease) [21]. In patients with Alzheimer's disease [19] or Lytico-bodig disease [21], MK immunoreactivity was found in senile plaques and neurofibrillary tangles. In addition, an increased expression of MK was found in astrocytes in rat models of cerebral ischemia [22]. It is not known, however, whether the expression of MK in the brain 
after the brain injury is a part of an endogenous repair process to prevent further damage in the CNS

The objectives of the present study are to determine whether intracerebroventricularly injected $\mathrm{MK}$ acts as an anticonvulsant and blocks KA-mediated neuronal cell death in hippocampus.

\section{Results}

\section{MK expression after seizures}

We first examined MK expression immunohistochemically in mouse hippocampus following $\mathrm{KA}$ injection. Injection of KA $(0.2 \mu \mathrm{g} /$ mouse $)$ to mice induced severe epileptiform seizures (mean score 4.2/maximum score 5.0). Basal level of MK immunoreactivity was found in hippocampal pyramidal neurons in control mouse brain injected with vehicle [phosphate-buffered saline (PBS)] (Figure 1A, top left panel), while in mouse injected with KA decreased MK expression was detected in pyramidal neurons (Figure 1A, bottom left panel; see arrows); Nissl staining of the adjacent sections confirmed that the cellular area of decreased $\mathrm{MK}$ immunoreactivity was associated with damaged pyramidal neurons (Figure 1A, bottom right panel; see arrows). Nissl staining in control animals receiving PBS injection showed no evident neuronal damage (Figure 1A, top right panel).

Interestingly, we have found the number of MK-positive cells markedly increased in stratum lacunosum moleculare (Slm) area of CA3 at 24-hr post-KA injection relative to PBS controls. Representative MK immunostaining in CA3 Slm is shown in Figure 1B (box denotes area of high magnification, top panel). In PBS-injected brain, only small number of MK-positive cells was observed (Figure 1B, middle panel). Following injection of KA, CA3 Slm area demonstrated an increase in the number of MK-positive cells (Figure 1B, bottom panel). Double-labeling immunofluorescence staining was then used to investigate $\mathrm{MK}$ expression in glial cells in KA-injected hippocampus. Representative double staining for $\mathrm{MK}$ (green staining, Figure 1C top panel) and GFAP (red staining, Figure 1C middle panel) showed that MK was expressed in GFAP-positive astrocytes; there was no MK expression in anti-complement receptor 3 (OX-42)-positive microglia (data not shown).

Quantification of MK expression data (expressed as area density of MK-positive cells, Figure 2) showed that KA injection significantly decreased $\mathrm{MK}$ immunoreactivity by $44 \%$ in SP area relative to PBS-injected control. However, the MK immunoreactivity in animals with KA injection markedly increased by $651 \%$ in Slm area as compared to PBS-injected control.

\section{Anticonvulsant effect of MK}

To further investigate the role of MK in KA-injected hippocampus, we examined the potential protective effects of MK against KA-induced seizures and neurotoxicity. Seizure onset was approximately $170 \mathrm{sec}$ following KA injec-

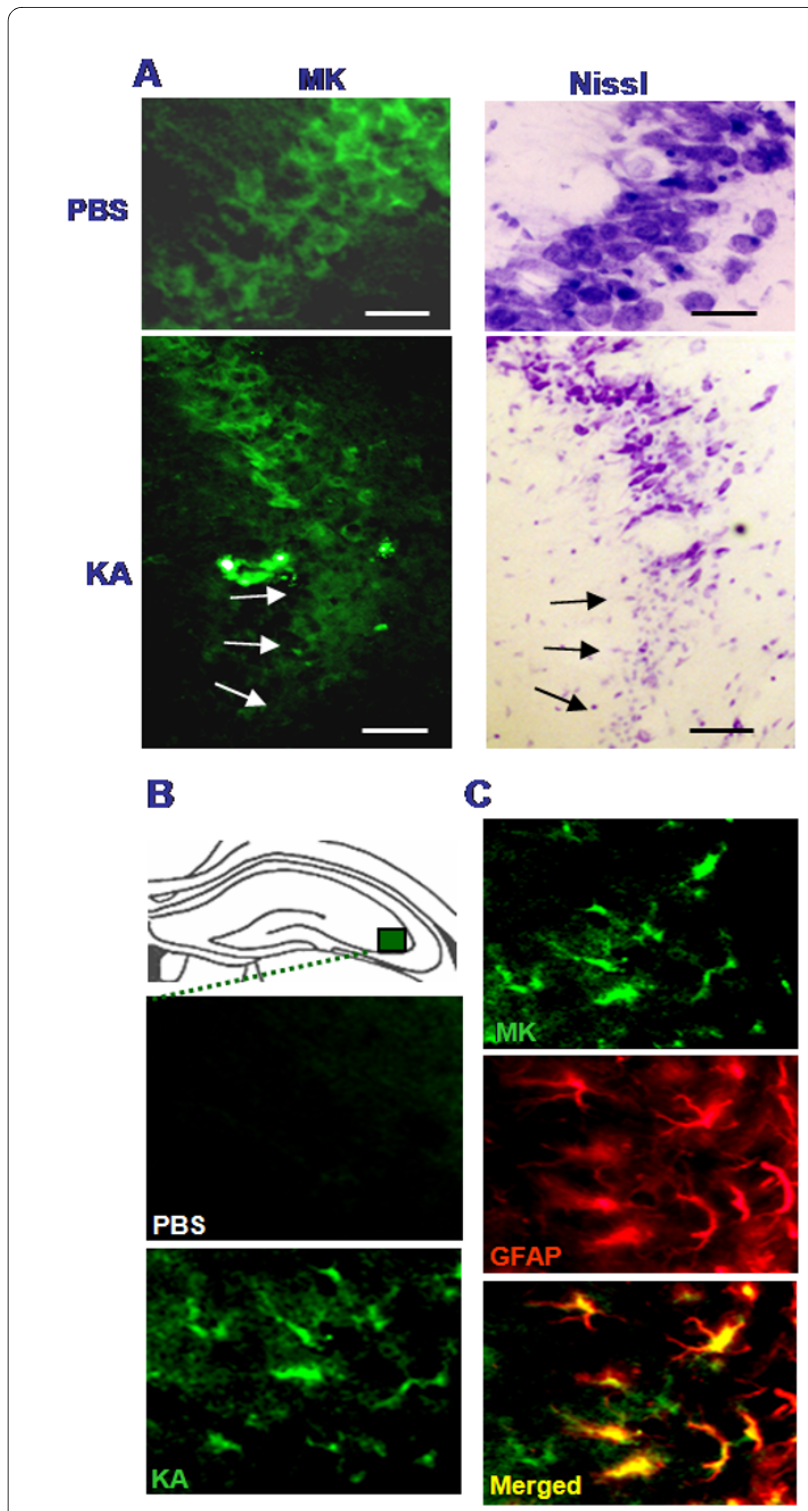

Figure $1 \mathrm{MK}$ expression in the hippocampus after KA injection. (A) Representative immunofluorescence images of MK immunoreactivity in hippocampal CA3 pyramidal neurons $24 \mathrm{hr}$ after PBS injection (top left panel) or KA $(0.2 \mu \mathrm{g} /$ mouse, bottom left panel). Adjacent hippocampal sections stained with Nissl staining (right panels) are shown here. Following KA treatment, cell death in CA3 pyramidal neurons is clearly visible (arrows). Scale bars: $20 \mu \mathrm{m}$ (top panels), $30 \mu \mathrm{m}$ (bottom panels). (B) Representative immunofluorescence images of MK-positive cells in CA3 SIm (striatum lacunosum moleculare) (box in top panel indicates region of interest) after injection with PBS (middle panel) or $\mathrm{KA}(0.2 \mu \mathrm{g} / \mathrm{mouse}$, bottom panel). (C) Double-labeling immunofluorescence staining of MK (green, top panel) with GFAP (red, middle panel) showing MK immunoreactivity is co-localized on GFAP-positive astrocytes in CA3 SIm (bottom panel). Scale bar: $20 \mu \mathrm{m}$.

tion, which lasted for mean 1,200 sec (Table 1). The intensity of seizures reached mean score 4.2 (maximum score 5.0) when the seizure activity was highest (5-10 min after KA challenge). Interestingly, seizure duration and 




Figure 2 Site-selective MK expression in the KA-damaged hippocampus. Bar graph shows the quantification of MK immunoreactivity in SP (stratum pyramidale) and SIm (stratum lacunosum moleculare) of hippocampal CA3 region $24 \mathrm{hr}$ after PBS or KA $(0.2 \mu \mathrm{g} /$ mouse) injection. Data are presented as mean \pm SEM. ${ }^{*} p<0.05$, as compared with PBS. One-way ANOVA, Student-Newman-Keuls multiple comparison test.

intensity were markedly reduced by co-administration of MK in a dose-dependent manner, although the time of onset time slightly delayed at a high dose $(0.4 \mu \mathrm{g} / \mathrm{mouse})$. Seizure duration and intensity were shortened and attenuated to $51.4-26.5 \%$ and $59.5-40.5 \%$ of the control levels by treatment with KA (0.1 - $0.4 \mu \mathrm{g} /$ mouse), respectively.

\section{Neuroprotective effect of MK}

Representative Nissl staining $24 \mathrm{hr}$ following KA injection showed considerable neuronal loss in the hippocampal subregions, $\mathrm{CA} 3$ and hilus of the dentate gyrus as compared to PBS control (Figure 3A, left and middle panels). MK treatment was effective in reducing neuronal loss in KAinjected hippocampus as shown in a representative finding in a high dose $(0.4 \mu \mathrm{g} /$ mouse) group (Figure $3 \mathrm{~A}$, right panels). The extent of degeneration of hippocampal neurons was quantified in hippocampal subregions, CA1, CA3, and hilus (Figure 3B). The number of hippocampal neurons was significantly reduced in CA3 (-81\%) and hilus (-85\%) by
KA exposure, whereas CA1 region (-19\%) was relatively spared when compared with PBS control. Co-application of MK increased survival of neurons in KA-injected hippocampus in a dose-dependent manner, leading to significant improvements at KA doses of 0.2 and $0.4 \mu \mathrm{g} /$ mouse.

Next we investigated the efficacy of MK to block the KA-induced cell death of GABAergic interneurons (GAD67-positive neurons). A previous study has reported that GAD67-positive interneurons are lost in the hippocampus in KA-injected animal model of excitotoxicity [23]. Animals receiving KA injection showed significant reduction in the number of GAD67-positive interneurons in subfields of CA1 (strata oriens $-80 \%$, strata pyramidale $-64 \%$, strata radiatum $-33 \%$ ), CA3 (strata oriens $-91 \%$, strata pyramidale $-78 \%$, strata radiatum $-62 \%$ ) and layers of dentate gyrus (molecular layer $-37 \%$, granule cell layer $-69 \%$, dentate hilus $-77 \%$ ) as compared to PBS control (Figures 4 and 5). Administration of $\mathrm{MK}(0.4 \mu \mathrm{g} /$ mouse $)$ reduced the cell loss in GAD67-positive neurons caused by KA injection and this neuroprotective effect of MK was evident in the subfields of CA3 and layers of dentate gyrus (Figure 4). Application of MK markedly increased survival of GAD67positive neurons in the strata pyramidale (68\% improvement), radiatum (45\% improvement) of CA3 and molecular layer $(27 \%$ improvement), granule cell layer $(29 \%$ improvement), and dentate hilus (33\% improvement) of dentate gyrus, whereas neurons in subfields of CA1 was not effectively preserved (Figure 5).

\section{Discussion}

The main finding of this study is that intracerebroventricular administration of MK conferred neuroprotection against KA-induced excitotoxic cell death of hippocampal neurons. Our results also demonstrated that $\mathrm{MK}$ was effective in attenuating KA-induced seizures and degeneration of GABAergic interneurons in hippocampus. The results from immunohistochemical staining showed that damaged pyramidal neurons in hippocampus are correlated with the decreased level of MK expression (Figure 1A). At present, it is not known whether this reduced level of MK immunoreactivity in neurons is causative factor for neuronal degen-

\section{Table 1: Effects of MK on KA-induced seizure activities}

\begin{tabular}{|c|c|c|c|}
\hline $\begin{array}{l}\text { Treatment } \\
\text { ( } \mu \mathrm{g} / \text { mouse) }\end{array}$ & $\begin{array}{l}\text { Latency to onset } \\
\text { (sec) }\end{array}$ & $\begin{array}{l}\text { Seizure duration } \\
\text { (sec) }\end{array}$ & $\begin{array}{l}\text { Seizure intensity } \\
\text { (maximum score } 5.0 \text { ) }\end{array}$ \\
\hline Vehicle & $0.0 \pm 0.0$ & $0.0 \pm 0.0$ & $0.0 \pm 0.0$ \\
\hline $\mathrm{KA}(0.2)$ alone & $169.9 \pm 38.8^{*}$ & $1,200.6 \pm 372.3^{*}$ & $4.2 \pm 0.1^{*}$ \\
\hline$+\mathrm{MK}(0.1)$ & $160.0 \pm 28.5$ & $616.7 \pm 50.3^{\#}$ & $2.5 \pm 0.3^{\#}$ \\
\hline$+\mathrm{MK}(0.2)$ & $172.8 \pm 33.8$ & $422.4 \pm 71.5^{\#}$ & $2.2 \pm 0.2^{\#}$ \\
\hline$+\mathrm{MK}(0.4)$ & $188.8 \pm 47.0$ & $318.6 \pm 31.7^{\#}$ & $1.7 \pm 0.3^{\#}$ \\
\hline
\end{tabular}

Data are presented as mean \pm SEM. ${ }^{*} p<0.05$, as compared with PBS. \# $p<0.05$, as compared with KA. Student's $t$-test. 

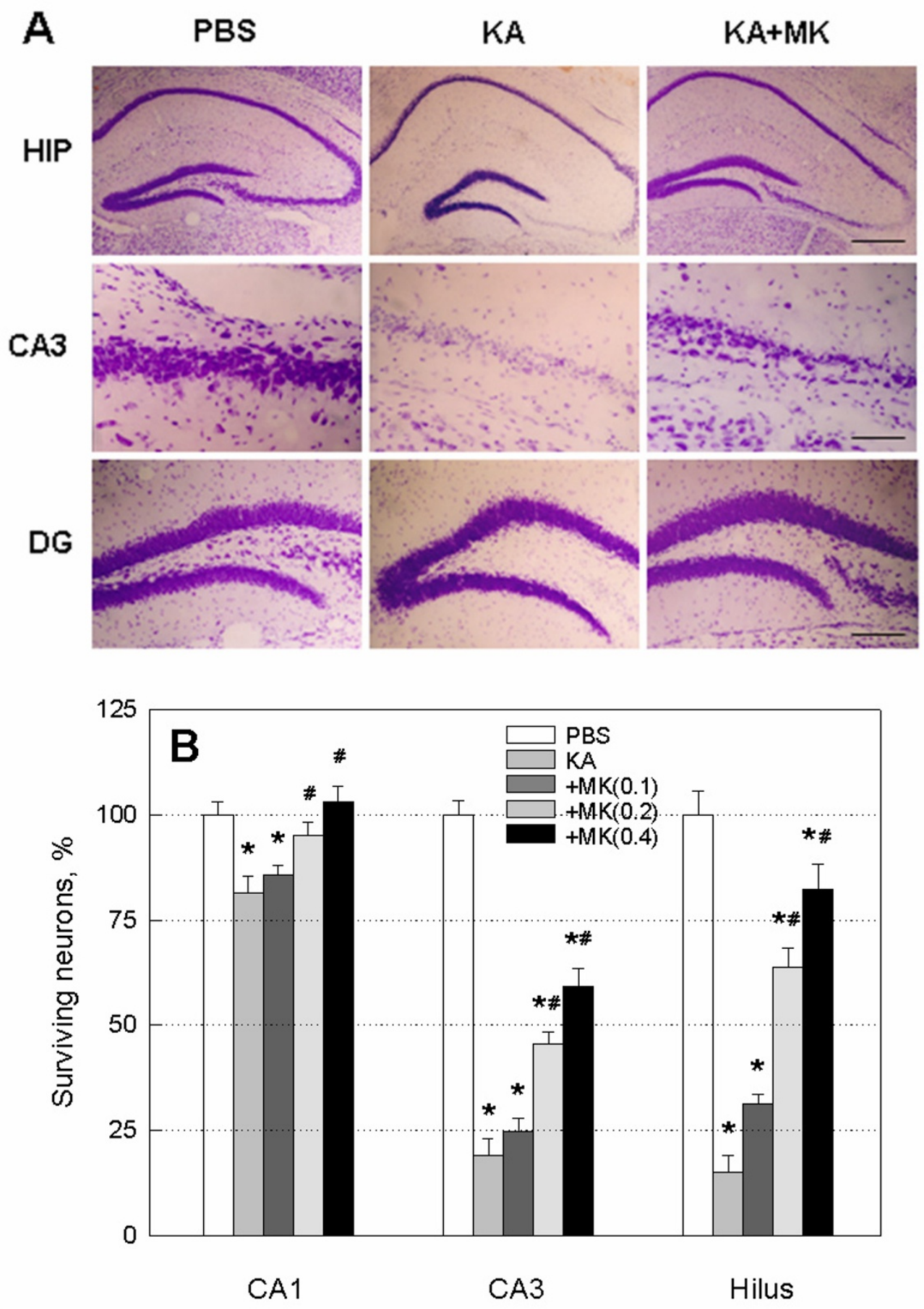

Figure 3 Effect of MK on hippocampal neuronal damage induced by KA injection. (A) Representative Nissl-stained sections from animals $24 \mathrm{hr}$ after PBS (left panels), KA (0.2 $\mathrm{mg} / \mathrm{mouse}$, middle panels) or KA plus MK $(0.4 \mu \mathrm{g} / \mathrm{mouse}$, right panels) injection. Top panels shows at low magnification of the hippocampus. Scale bar: $400 \mu \mathrm{m}$. Middle and bottom panels show at high magnification of CA3 and dentate gyrus (DG) subregions of hippocampus. Scale bars: $200 \mu \mathrm{m}$. Note that the damaged areas induced by KA are indicated by absence of Niss--positive neurons. (B) Quantification of undamaged Niss--positive neurons for the hippocampal subregions CA1 and CA3, and hilus of dentate gyrus with different MK doses (0.1 -- $0.4 \mu \mathrm{g} /$ mouse). Data are presented as mean \pm SEM. ${ }^{*} p<0.05$, as compared with PBS. \# $p<0.05$, as compared with KA. One-way ANOVA, Student-NewmanKeuls multiple comparison test. 


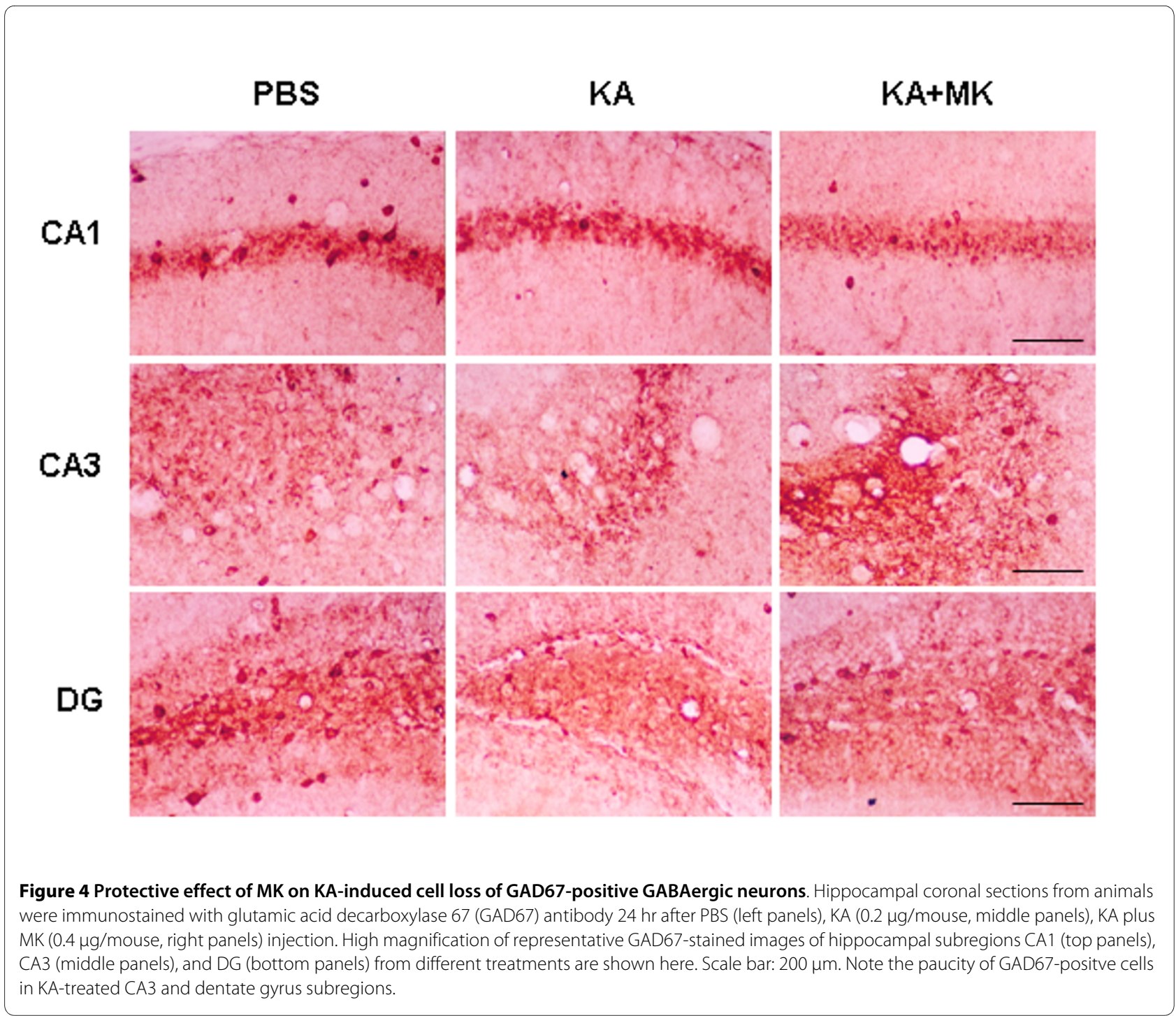

eration induced by KA injection. However, previous in vivo studies have demonstrated that MK is an effective neuroprotective agent in reducing retinal degeneration caused by excessive light [24] and decreasing hippocampal neuronal death in ischemic gerbil brain [25]. Furthermore, MK knock-out mice displayed altered expression of calciumbinding protein in hippocampus and defective working memory [26]. In view of these observations, it is possible that MK is involved in the regulation of endogenous neuroprotective process against externally applied injury or disease.

We have found that the level of MK immunoreactivity markedly increased in astrocytes after KA injection (Figure 1C). In a previous report, we have demonstrated rapid activation of astrocytes in hippocampal alveus and fimbria, strata oriens and lacunosum moleculare as well as dentate hilus as early as $1 \mathrm{hr}$ following diisopropyl fluorophosphate (DFP)-induced seizures and neuronal injury [27]. These observations suggest that the activated astrocytes serve as an important source of MK in response to excitotoxicity. Important findings in the present study are therapeutic and neuroprotective effects of $\mathrm{MK}$ on KA-induced neuronal injury in CA3 and dentate hilus of hippocampus (Figure 3). Our results are in good agreement with a previous study of neuroprotective effect of $\mathrm{MK}$ in transient forebrain ischemia when given immediately before middle cerebral artery occlusion [25]. Previous studies have demonstrated that KA-injected brain show dramatic reduction in the number of GAD-positive interneurons [7,23,28], even in CA1 statum oriens and alveus where pyramidal neurons are relatively spared [Figures 3-5][29], which can cause abnormal functional inhibition of neuronal circuitry leading to hippocampal hyperexcitability $[2,7]$. Thus, it is noteworthy that icv application of MK blocks the degeneration of GAD67-positive interneurons, especially in the stratum pyramidale and radiatum of $\mathrm{CA} 3$, and the molecular layer, 

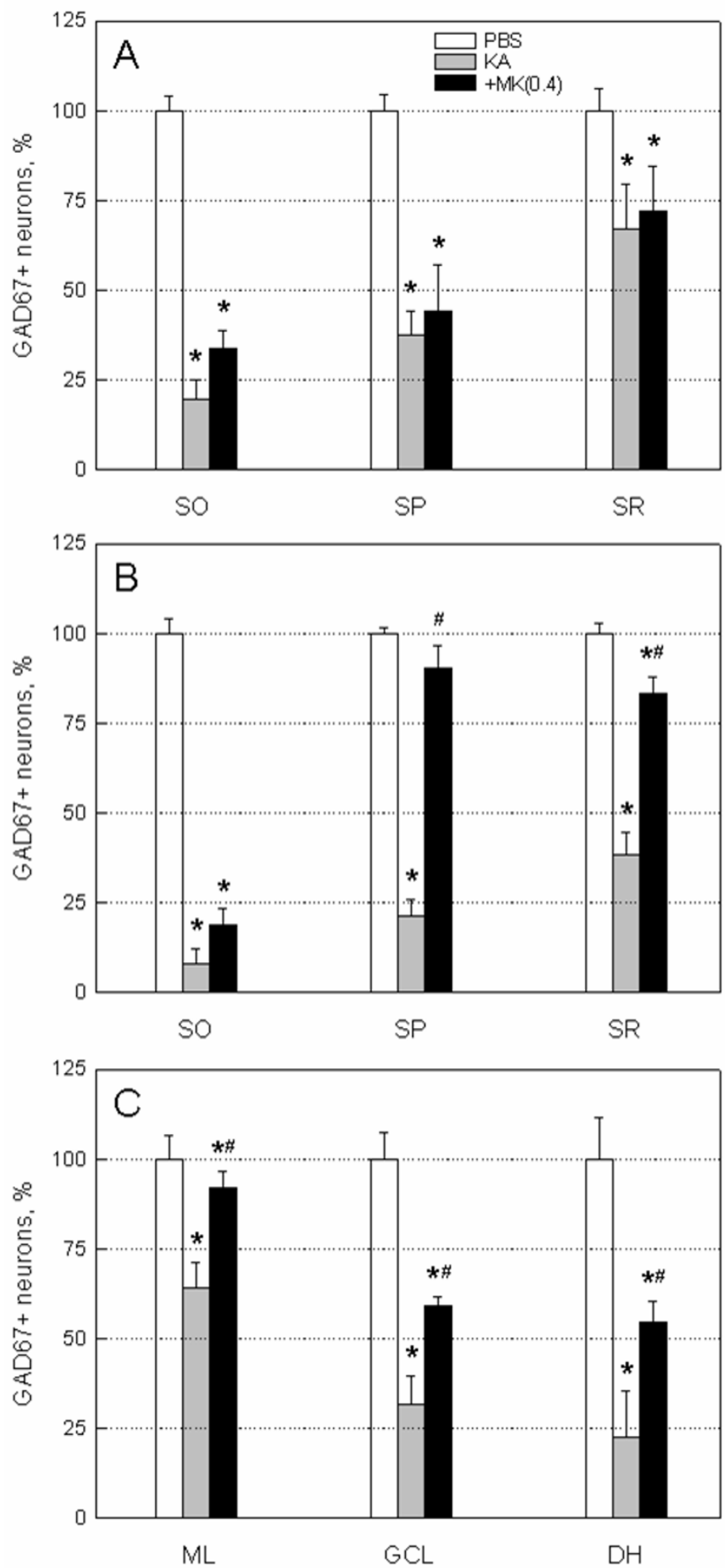

Figure 5 Protective effect of MK on KA-induced GABAergic neuronal cell death. Numbers of GAD67+ neurons were counted in subregions of

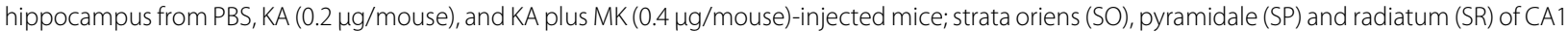
(A) and CA3 (B) and all layers of dentate gyrus (C) (ML; molecular layer, GCL; granule cell layer, DH; dentate hilus). Data are presented as mean \pm SEM. ${ }^{*} p<0.05$, as compared with PBS. \# $p<0.05$, as compared with KA. One-way ANOVA, Student-Newman-Keuls multiple comparison test. 
granule cell layer and hilus of dentate gyrus as caused by KA injection (Figures 4 and 5).

The majority of the animals experienced stage $4 / 5$ seizure severity after KA injection. A previous study has demonstrated in KA-injected rat epilepsy model that dizocilpine (MK-801) inhibited hippocampal neuronal loss without blocking seizure development [30]. In addition, several studies in animal model of TLE have also shown that significant neuronal loss is not necessarily a prerequisite for the development of seizures [31,32]. Such results imply that the mechanism of MK action in TLE model is different from that of MK-801, an NMDA antagonist. Our results show that MK-induced neuroprotection against KA toxicity is primarily associated with moderation of seizure activity (Table 1). It is believed that MK plays a role as an anticonvulsant directly (Table 1) or indirectly by preserving an inhibitory amino acid (GABA) system including GAD67positive interneurons (Figures 4 and 5). Furthermore, it is possible to speculate that MK could be a neurotrophic factor, especially for GAD67-positive interneurons or astrocytes expressing high levels of MK (Figure 1) which may contribute to early cessation of seizure and control of its recurrence.

The exact mechanism of the neuroprotective activity of MK remains to be further clarified but activation of gene product(s) associated with apoptosis by MK may provide some answers. For instance, previous studies have shown that MK inhibits apoptotic process by up-regulation of bcl2 expression [33] and by inhibition of caspase- 3 activation [34]. Thus, it is possible that the MK-mediated neuroprotective mechanism involves activation of signal transduction pathways involved in regulation of apoptotic cell death.

\section{Conclusions}

The results of the present study demonstrate that the administration of $\mathrm{MK}$ produces a significant neuroprotective effect against KA-induced neuronal loss in mouse model of epilepsy. Additional studies of MK to elucidate their neuroprotective activity in animal models of brain injury and neurodegeneration such as Parkinson's disease, Huntington's disease, amyotropic lateral sclerosis, stroke or spinal cord injury should prove $\mathrm{MK}$ as a member of neurotrophic factors that are valuable in providing effective treatment for patients with various neurological disorders.

\section{Methods}

\section{Treatment and seizure monitoring}

Male C57BL/6 mice ( $\mathrm{n}=8$ /group) weighing 25 - 30 g were used for the experiments. The animals were housed in a temperature- and humidity-controlled room that was kept on an alternating 2-hr light/dark schedule. Food and water were available ad libitum throughout the experiments. All animal experiments were conducted in accordance with the on Animal Care Committee of the University of British Columbia.

Animals were anesthetized with intraperitoneal injection of chloral hydrate $(7 \%, 0.1 \mathrm{~mL} / \mathrm{kg})$ and then mounted in a stereotaxic apparatus (David Kopf Instruments, Tujunga, CA). KA $(0.2 \mu \mathrm{g} / 0.4 \mu \mathrm{L}$ PBS/mouse; Sigma, St. Louis, $\mathrm{MO})$ was injected into the right lateral cerebroventricle at the coordinate (AP, -2.0; ML, -2.9; DV, -3.8) using a $10 \mu \mathrm{L}$ Hamilton syringe fitted with $26 \mathrm{G}$ needle at a rate of $0.1 \mu \mathrm{L} /$ $\mathrm{min}$. The needle was left in place for $5 \mathrm{~min}$. MK $(0.1,0.2$ or $0.4 \mu \mathrm{g} / 0.4 \mu \mathrm{L}$ PBS; LG Biotech, Daejon, Korea) was unilaterally co-injected with KA into the right lateral cerebroventricle. For sham-operated animals, the same volume of 0.1 M PBS was stereotaxically injected into the same coordinates described above. Wounds were sutured, and animals were allowed to recover and then returned to their cages. After KA injection, each animal was placed in a Plexiglas cylinder and their seizure behaviors - latency to onset, duration, and intensity - were recorded for a period of $120 \mathrm{~min}$ in a blind manner. Seizure intensity was scored with a slight modification from a previous scoring system [35] as followed: stage 1, immobilization and staring; stage 2, head nodding; stage 3, rearing accompanied by forelimb clonus and wet dog shakes; stage 4, falling and wobbling; stage 5, jumping, circling, or rolling. The starting time of head nodding was considered onset time of seizures, because the starting of immobilization and staring behaviors (stage 1) was not clear.

\section{Immunohistochemistry}

The animals were anesthetized and transcardially perfused with $50 \mathrm{~mL}$ cold saline followed by $100 \mathrm{~mL}$ of $4 \%$ paraformaldehyde in $0.1 \mathrm{M}$ phosphate buffer ( $\mathrm{pH} 7.4) 24 \mathrm{hr}$ after KA administration. The brains were removed from the skull and post-fixed in 4\% paraformaldehyde for $24 \mathrm{hr}$, followed with cryoprotection in $30 \%$ sucrose in phosphate buffer for 2 days. Serial coronal sections at $30 \mu \mathrm{m}$ were prepared on a cryostat (CM 1900; Leica, Heerbrugg, Switzerland). Free-floating sections were prepared from the brains of PBS, KA, and KA plus MK-injected mice. For single immunofluorescence staining, brain sections were incubated in PBS containing 5\% normal goat serum and $0.2 \%$ Triton X-100 for $30 \mathrm{~min}$ at room temperature (RT), and then incubated overnight with rabbit anti-MK antibody (1:500, kindly provided by Dr. T. Muramatsu, Nagoya University, Japan). Sections were then incubated with Alexa Fluor 488-conjugated goat anti-rabbit IgG (1:200; Molecular Probes, Eugene, OR) at RT for $2 \mathrm{hr}$ in the dark. Sections were washed in phosphate buffer, mounted on slides. Nissl staining was also performed on slide-mounted brain sections with $0.1 \%$ cresyl violet (Sigma) for the evaluation of hippocampal neuronal loss.

For GAD67 immunohistochemical staining, all incubation solutions did not contain Triton X-100. The brain sec- 
tions were briefly quenched with $3 \% \mathrm{H}_{2} \mathrm{O}_{2}$ in PBS for 10 min and incubated with 5\% normal goat serum for $30 \mathrm{~min}$. The sections were incubated overnight at $4^{\circ} \mathrm{C}$ with rabbit anti-GAD67 (1:1000; Chemicon, Temecula, CA). The sections were then incubated for $1 \mathrm{hr}$ with biotinylated antirabbit IgG (1:200; Vector, Burlingame, CA), followed by incubation with avidin-biotin complex (1:200, Vector) for 1 $\mathrm{hr}$ and then visualized with $0.05 \% 3,3^{\prime}$-diaminobenzidine (Sigma) and $0.003 \% \mathrm{H}_{2} \mathrm{O}_{2}$. Negative control sections were prepared for immunohistochemical staining in an identical manner except the primary antibodies were omitted.

\section{Double-labeling immunofluorescence microscopy}

Free floating sections were incubated in PBS containing 3\% normal goat serum and $0.3 \%$ Triton X-100 for $30 \mathrm{~min}$ at room temperature (RT). Brain sections were incubated for $48 \mathrm{hr}$ at $4^{\circ} \mathrm{C}$ in a mixture of two primary antibodies: MK $(1: 100)$ in combination with mouse anti-GFAP (1:500; Sigma) or mouse OX-42 (1:200; Serotec, Oxford, UK). Sections were then incubated in a mixture of Alexa Fluor 488-conjugated goat anti-rabbit IgG (1:200; Molecular Probes) and Alexa Fluor 594-conjugated goat anti-mouse IgG (1:200; Molecular Probes) at RT for $2 \mathrm{hr}$ in the dark. Processed sections were mounted on gelatin-coated slides, coversliped and examined under a Zeiss Axioplan-2 microscope.

\section{Quantitative analysis}

Five coronal hippocampal sections (at the level of the injection site and spaced $60 \mu \mathrm{m}$ from each other) were used for immunohistochemical analysis. All quantitative analyses were performed in a blind manner. To ensure consistency in tissue sampling, matched hippocampal sections (based on anatomical landmark) were always processed throughout the experiments. Digitized images of stained sections were acquired using a Zeiss Axioplan-2 microscope equipped with a DVC camera (Diagnostic Instruments, Sterling Heights, MI). These images were then analyzed using Northern Eclipse software (Empix Imaging, Mississauga, ON, Canada). Three microscopic fields within SP (stratum pyramidale) and Slm (stratum lacunosum moleculare) areas in CA3 were selected (magnification of $\times 40$ ) in MK-stained sections. MK immunoreactivity was then measured and expressed as area density of MK (the fraction of the total given area occupied by MK-positive cells). Three microscopic fields (magnification at $\times 40$ ) in CA1, CA3 and hilus region were counted for Nissl-stained undamaged neurons in each coronal brain section. Neurons are identified by dark nucleoli within lightly stained nuclei. GAD67-positive neurons were quantified in CA1 and CA3 subfields (strata oriens, radiatum, and pyramidale) and dentate gyrus layers (dentate hilus, granule cell layer, and molecular layer). These regions were established according to the atlas of Paxinos \& Watson [36]. Number of Nissl- and GAD67-pos- itive neurons was expressed as a percentage of the PBSinjected control sections.

\section{Statistical analysis}

Data are presented as means SEM. The statistical significance was determined by one-way ANOVA and StudentNewman-Keuls test or Student's $t$-test using the StatView program (Abacus Concepts, Berkeley, CA). $p$ values $<0.05$ were considered to be statistically significant.

\section{Authors' contributions}

YBK and JKR designed the study, carried out animal experiments and drafted the manuscript. HJL, IJL, and DP performed histology and histochemistry, and MCL participated in the design of the study and histological analysis. SUK conceived of the study, participated in its design and coordination, and drafted the manuscript. All authors read and approved the final manuscript.

\section{Acknowledgements}

This work was supported by grants from the National R\&D Program for Cancer Control, Korean Ministry of Health and Welfare and the Canadian Myelin

Research Initiative.

\section{Author Details}

'Division of Neurology, Department of Medicine, UBC Hospital, University of British Columbia, Vancouver, Canada, ${ }^{2}$ College of Veterinary Medicine, Chungbuk National University, Cheongju, Korea, ${ }^{3}$ Medical Research Institute, Chung-Ang University College of Medicine, Seoul, Korea, ${ }^{4}$ Department of Physiology, Chung-Ang University College of Medicine, Seoul, Korea and 5Department of Pathology, Chonnam National University Medical School, Gwangju, Korea

Received: 31 August 2009 Accepted: 26 March 2010 Published: 26 March 2010

\section{References}

1. Bruton CJ: The neuropathology of temporal lobe epilepsy. New York, Oxford University Press; 1988.

2. Sloviter RS: The functional organization of the hippocampal dentate gyrus and its relevance to the pathogenesis of temporal lobe epilepsy. Ann Neurol 1994, 35:640-654.

3. Nadler JV, Perry BW, Cotman CW: Intraventricular kainic acid preferentially destroys hippocampal pyramidal cells. Nature 1978 271:676-677.

4. Franck JE: Dynamic alterations in hippocampal morphology following intra-ventricular kainic acid. Acta Neuropathol 1984, 62:242-253.

5. Ben-Ari Y: Limbic seizures and brain damage produced by kainic acid: Mechanisms and relevance to human temporal lobe epilepsy. Neuroscience 1985, 14:375-403.

6. Sperk G: Kainic acid seizures in the rat. Prog Neurobio/ 1994, 42:1-32.

7. Shetty AK, Turner DA: Fetal hippocampal grafts containing CA3 cells restore host hippocampal glutamate decarboxylase-positive interneuron numbers in a rat model of temporal lobe epilepsy. $J$ Neurosci 2000, 20:8788-8801.

8. Gall CM, Isackson PJ: Limbic seizures increase neuronal production of messenger RNA for nerve growth factor. Science 1989, 245:758-761.

9. Mathern GW, Babb TL, Micevych PE, Blanco CE, Pretorius JK: Granule cell mRNA levels for BDNF, NGF, and NT-3 correlate with neuron losses or supragranular mossy fiber sprouting in the chronically damaged and epileptic human hippocampus. Mol Chem Neuropathol 1997, 30:53-76.

10. Rauvala $\mathrm{H}$ : An 18-kd heparin-binding protein of developing brain that is distince tfrom fibroblast growth factors. EMBO J 1989, 8:2933-2941.

11. Muramatsu $H$, Shirahama $H$, Yonezawa S, Maruta $H$, Muramatsu T: Midkine, a retinoic acid-inducible growth/differentiation factor: immunochemical evidence for the function and distribution. Dev Biol 1993, 159:392-402.

12. Kadomatsu K, Tomomura M, Muramatsu T: cDNA cloning and sequencing of a new gene intensely expressed in early differentiation stages of embryonal carcinoma cells and in midgestation period of 
mouse embryogenesis. Biochem Biophys Res Commun 1988, 151:1312-1318.

13. Muramatsu $\mathrm{H}$, Muramatsu T: Purification of recombinant midkine and examination of its biological activities: functional comparison of new heparin binding factors. Biochem Biophys Res Commun 1991 177:652-658.

14. Michikawa M, Kikuchi S, Muramatsu H, Muramatsu T, Kim SU: Retinoic acid responsive gene product, midkine, has neurotrphic functions in mouse spinal cord and dorsal root ganglion neurons in culture. Neurosci Res 1993, 35:530-539.

15. Satoh J, Muramatsu H, Moretto G, Chang HJ, Kim ST, Cho JM, Kim SU: Midkine that promotes survival of fetal human neurons is produced by fetal astrocytes in culture. Brain Res Dev Brain Res 1993, 75:201-205.

16. Kikuchi S, Muramatsu H, Muramatsu T, Kim SU: Midkine, a novel neurotrophic factor, promotes survival of mesencephalic neurons in culture. Neuroscilett 1993, 160:9-12.

17. Kikuchi-Horie K, Kawakami E, Kamata M, Wada M, Hu JG, Ohara K, Watabe $\mathrm{K}$, Oyanagi $\mathrm{K}$ : Distinctive expression of midkine in the repair period of rat brain during neurogenesis: immunohistochemical and immunoelectron microscopic observations. J Neurosci Res 2004, 75:678-687.

18. Ratovitski EA, Kotzbauer PT, Milbrandt J, Lowenstein CJ, Burrow CR: Midkine induces tumor cell proliferation and binds to a high affinity signaling receptor associated with JAK tyrosine kinases. J Bio/ Chem 1998, 273:3654-3660.

19. Yasuhara O, Muramatsu H, Kim SU, Muramatsu T, Maruta H, McGeer PL: Midkine, a novel neurotrophic factor, is present in senile plaques of Alzheimer disease. Brain Res Dev Brain Res 1993, 192:246-251.

20. Yoshida Y, Goto M, Tsutsui J: Midkine is present in the early stage of cerebral infarct. Brain Res Dev Brain Res 1995, 85:25-30.

21. Yasuhara O, Schwab C, Matsuo A, Kim SU, Steele JC, Akiguchi I, Kimura H, McGeer EG, McGeer PL: Midkine-like immunoreactivity in extracellular neurofibrillary tangles in brains of patients with parkinsonismdementia complex of Guam. Neurosci Lett 1996, 205:107-110.

22. Mochizuki R, Takeda A, Sato N, Kimpara T, Onodera H, Itoyama Y, Muramatsu T: Induction of midkine expression in reactive astrocytes following rat transient forebrain ischemia. Exp Neurol 1998, 149:73-78.

23. Buckmaster PS, Jongen-Relo AL: Highly specific neuron loss preserves lateral inhibitory circuits in the dentate gyrus of kainate-induced epileptic rats. J Neurosci 1999, 19:9519-9529.

24. Unoki K, Ohba N, Arimura H, Muramatsu H, Muramatsu T: Rescue of photoreceptors from the damaging effects of constant light by midkine, a retinoic acid-responsive gene product. Invest Ophthal Vis Sci 1994, 35:4063-4068

25. Yoshida Y, Ikematsu S, Moritoyo T, Goto M, Tsutsuji J, Sakuma S, Osame M, Muramatsu T: Intraventricular administration of the neurotrophic factor midkine ameliorates hippocampal delayed neuronal death following transient forebrain ischemia in gerbils. Brain Res 2001, 894:46-55.

26. Nakamura E, Kadomatsu K, Yuasa S, Muramatsu H, Nabeshima T, Fan QW, Ishiguro K, Igakura T, Matsubara S, Kaname T, Horiba M, Saaito H, Muramatsu T: Disruption of the midkine gene (Mdk) resulted in altered expression of a calcium binding protein in the hippocampus of infant mice and their abnormal behavior. Genes Cells 1998, 12:811-822.

27. Kim Y-B, Hur G-H: Seizure-related encephalopathy in rats intoxicated with diisopropylfluorophosphate. J Toxicol Pub Health 2001, 17:73-82.

28. Houser CR, Esclapez M: Vulnerability and plasticity of the GABA system in the pilocarpine model of spontaneous recurrent seizures. Epilepsy Res 1996, 26:207-218.

29. Morin F, Beaulieu C, Lacaille J-C: Selective loss of GABA neurons in area CA1 of the rat hippocampus after intraventricular kainite. Epilepsy Res 1998, 32:363-369

30. Brandt C, Potschka H, Löscher W, Ebert U: N-methyl-D-aspartate receptor blockade after status epilepticus protects against limbic brain damage but not against epilepsy in the kainate model of temporal lobe epilepsy. Neuroscience 2003, 118:727-740.

31. Bertram EH, Scott C: The pathological substrate of limbic epilepsy: neuronal loss in the medial dorsal thalamic nucleus as the consistent change. Epilepsia 2000, 41(Suppl 6):S3-S8

32. Raol YS, Budreck EC, Brooks-Kayal AR: Epilepsy after early-life seizures can be independent of hippocampal injury. Ann Neurol 2003, 53:503-511.
33. Qi M, Ikematsu S, Ichihara-Tanaka K, Sakuma S, Muramatsu T, Kadomatsu K: Midkine rescues Wilms' tumor cells from cisplatin-induced apoptosis: regulation of $\mathrm{BCl}-2$ expression by midkine. J Biochem 2000, 127:269-277.

34. Owada K, Sanjo N, Kobayashi T, Mizusawa H, Muramatsu H, Muramatsu T, Michikawa M: Midkine inhibits caspase-dependent apoptosis via the activation of mitogen-activated protein kinase and phosphatidylinositol 3-kinase in cultured neurons. J Neurochem 1999 73:2084-2092

35. Benkovic SA, O'Callaghan JP, Miller DB: Sensitive indicators of injury reveal hippocampal damage in C57BL/6J mice treated with kainic acid in the absence of tonic-clonic seizures. Brain Res 2004, 1024:59-76.

36. Paxinos $G$, Watson $C$ : The rat brain in stereotaxic coordinates. 5th edition. Elsevier Academic Press; 2005.

doi: $10.1186 / 1471-2202-11-42$

Cite this article as: Kim et al., Midkine, heparin-binding growth factor, blocks kainic acid-induced seizure and neuronal cell death in mouse hippocampus BMC Neuroscience 2010, 11:42

\section{Submit your next manuscript to BioMed Central and take full advantage of:}

- Convenient online submission

- Thorough peer review

- No space constraints or color figure charges

- Immediate publication on acceptance

- Inclusion in PubMed, CAS, Scopus and Google Scholar

- Research which is freely available for redistribution
C Biomed Central 